\title{
Aeroelastic Topology Optimization of Blade-Stiffened Panels
}

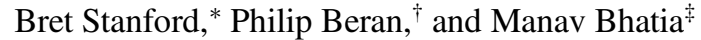 \\ U.S. Air Force Research Laboratory, Wright-Patterson Air Force Base, Ohio 45433
}

DOI: $10.2514 / 1 . C 032500$

\begin{abstract}
Metallic blade-stiffened panels are optimized for various eigenvalue metrics of interest to the aerospace community. This is done via solid isotropic material with penalization-based topology optimization: the stiffeners are discretized into finite elements, and each element is assigned a design variable, which may vary from 0 (void) to 1 (solid). A known issue with eigenvalue-based optimization is discontinuities due to mode switching, which may be avoided through a series of eigenvalue separation constraints, or (more challenging, but less restrictive) a bound method with mode tracking. Both methods are demonstrated to obtain optimal stiffener topologies for panel buckling, but only the former is used for aeroelastic panel-flutter problems. Satisfactory flutter optimal results are obtained, but the work concludes with a discussion of the challenges associated with the use of a bound method for aeroelastic problems, with specific complications posed by the advent of hump modes.
\end{abstract}

\section{Introduction}

$\mathbf{T}$ HIS work considers the topological optimization of bladestiffened-panel structures for aerospace applications. A recent overview of stiffened plates and shells is given by Bedair [1], who discusses the important role these structures can play across a broad range of engineering applications, including aircraft. We are specifically concerned with panels subjected to a high-speed flow over their upper surface and high temperatures from the concomitant aerodynamic heating and/or thermal radiation (specifically, the panel buckling that may result from such loading). Topology optimization can be used to obtain the underlying support structure (i.e., stiffener reinforcement), which provides a suitable compromise between structural mass, buckling, and aeroelastic flutter metrics. Two main issues are discussed here. The first issue is the topological parameterization of the underlying support structure; the second details methods to handle eigenvalue-migration metrics (found in flutter problems) during topology optimization.

Concerning first the issue of structural parameterization, many papers and commercially available software packages exist for stiffened-panel design, under the assumption that the basic topology or layout of the underlying stiffeners is largely predetermined. Typical design variables may include stiffener dimensions, stiffener spacing, and basic stiffener concepts (i.e., type of stiffener), as reviewed in [2]. Far fewer papers exist in the literature, which relax this assumption. One option is to discretize the design domain beneath the panel face sheet into brick finite elements, and assign a density variable to each, as done in [3]. This is very expensive, however, as a large finite element model and many design variables are needed to obtain a refined topological geometry.

Alternatively, a variable-thickness panel optimization may be undertaken, and areas of high thickness may be subsequently identified as potential stiffener locations (see Luo and Gea [4], Lam and Santhikumar [5], and Khosravi et al. [6], for example). This procedure may fail if the variable-thickness design does not identify a riblike thickness pattern, but instead converges to a structure with

Presented as Paper 2013-1871 at the 54th AIAA/ASME/ASCE/AHS/ASC Structures, Structural Dynamics, and Materials Conference, Boston, MA, 8 11 April 2013; received 27 June 2013; accepted for publication 22 October 2013; published online 21 March 2014. This material is declared a work of the U.S. Government and is not subject to copyright protection in the United States. Copies of this paper may be made for personal or internal use, on condition that the copier pay the $\$ 10.00$ per-copy fee to the Copyright Clearance Center, Inc., 222 Rosewood Drive, Danvers, MA 01923; include the code 1542-3868/14 and \$10.00 in correspondence with the CCC.

*Research Engineer, Universal Technology Corporation; bretkennedystanford@gmail.com. Member AIAA.

†Principal Research Aerospace Engineer; philip.beran@wpafb.af.mil. Associate Fellow AIAA.

*Research Engineer, Universal Technology Corporation; manav.bhatia. ctr@wpafb.af.mil. Member AIAA. large lobes of concentrated mass (as may be expected for vibration problems). Other authors have limited the structural parameterization specifically to the desired stiffener geometry, and used branching tree models (Ding and Yamazaki [7]) or embedded fiber introduction (Bojczuk and Szteleblak [8]). Although not yet used for stiffenedplate layout design, the cellular-based topology-optimization scheme developed by Pedro and Kobayashi [9] may be a suitable method as well.

Having located the optimal stiffener layout, or ground structure, the internal topology of each stiffener may then be determined using a standard solid isotropic material with penalization (SIMP)-based approach, in which each stiffener is discretized into shell finite elements, and the constitutive material tensor for each is attached to a design variable $[10,11]$. For this work, the initial ground structure of the stiffeners is simply predetermined. A finely detailed stiffenerlayout pattern may provide the optimizer with enough latitude to make substantial changes to the structure. If a material is not needed in an area where a stiffener has been allocated, the SIMP-based optimizer can simply remove all of the material from this potential rib. An example of this process is shown graphically in Fig. 1, for two different stiffener layouts. More sophisticated optimization methods (coupling the evolutionary approach of [9] for general layout design to the SIMP-based approach for internal topologies, using a bilevel method, for example) may be considered in the future.

The second issue listed previously is eigenvalue migration. It is well known that optimization problems built upon eigenvalue metrics may have discontinuities in the design space due to mode crossings. This issue becomes more strenuous with aeroelastic flutter problems, in which the flutter point is defined as the flight speed at which one of the eigenvalues becomes dynamically unstable [12] (positive real part). This destabilization is typically, but not always, associated with the coalescence of the imaginary parts (i.e., frequencies) of two eigenvalues. Between consecutive design iterations, two detrimental scenarios may occur. First, the underlying flutter mechanism may switch, but the new flutter point occurs at a very similar flight speed to the old one. This is a $C^{1}$ discontinuity in the design space: at best, it will slow down the convergence of the gradient-based optimizer. This change is shown graphically in Fig. 2 , in which $\lambda$ is an aerodynamic pressure parameter, and $g$ is the real part of each eigenvalue (aeroelastic damping).

Alternatively, the flutter mechanism may switch in such a way that the flutter speed drastically decreases. This is seen in Fig. 3 , in which the lower flutter point manifests itself through a hump mode. This is a severe $C^{0}$ discontinuity in the design space, which typically renders the gradient-based optimizer entirely ineffective. Langthjem and Sugiyama [13] and Odaka and Furuya [14] have used a series of frequency-separation constraints to prevent the critical flutter mechanism from switching during the design process. For every value of $\lambda$ between 0 (wind off) and the flutter point, the imaginary portions (frequencies) of modes 2 and 3 must always be separated by 


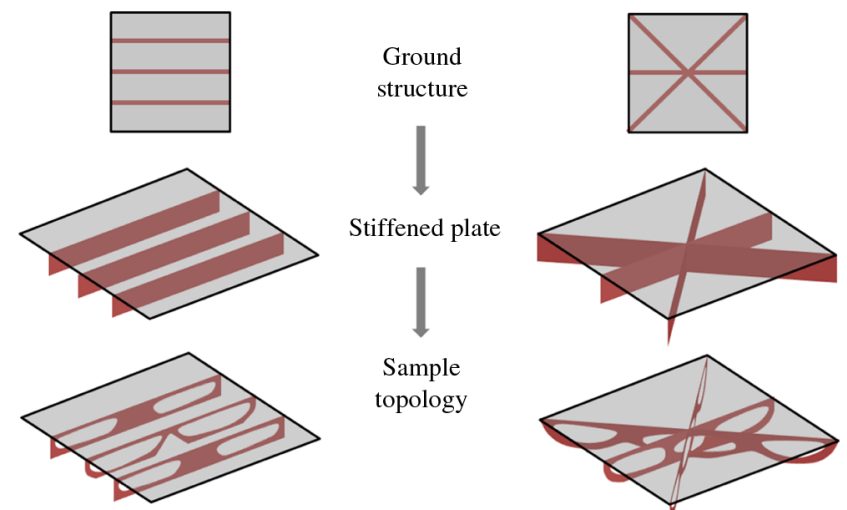

Fig. 1 Typical stiffener topologies that may arise for two different ground structures.
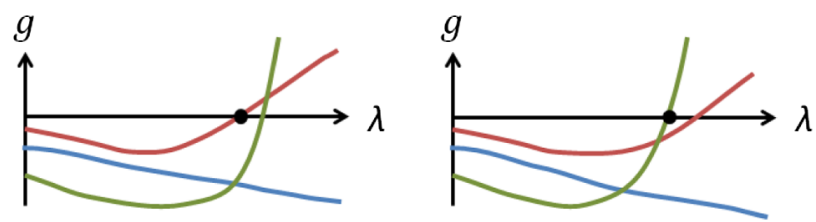

Fig. 2 Eigenvalue migration (real part): change in flutter mechanism with benign change in flutter speed from one design iterate (left) to the next (right).
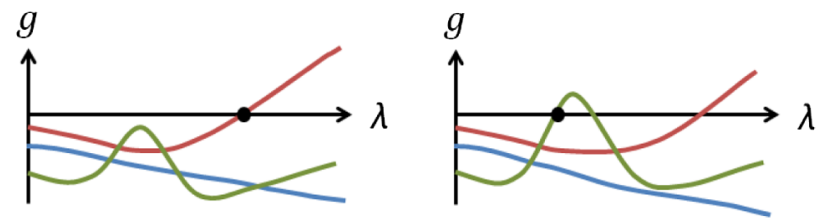

Fig. 3 Eigenvalue migration (real part): change in flutter mechanism with severe change in flutter point from one design iterate (left) to the next (right).

some buffer. The same constraint is imposed between modes 3 and 4 , modes 4 and 5, etc., up to the number of eigenvalues retained in the analysis. This is shown graphically on the left of Fig. 4 , in which $\omega$ is the imaginary part of each eigenvalue.

Frequency separation is motivated by the frequency coalescence discussed previously: keeping the frequencies separated can prevent the higher-mode bifurcations seen in Figs. 2 and 3 , and the associated discontinuities. For this work, however, flutter was observed without a strict coalescence of modes, particularly for cases with strong preflutter aeroelastic damping (which is entirely neglected in [14]). Mode switching could still occur in spite of large frequency separations, slowing or preventing optimization convergence. A second idea is discussed by Haftka [15], who enforces a series of critical-damping constraints. For every value of $\lambda$ between 0 (wind off) and the flutter point, the real portions (damping) of modes 2, 3, etc. must always be less than some threshold $g_{\text {cr }}$ (shown by a dashed line on the right of Fig. 4). In the example of Fig. 4, the satisfaction of this constraint is preventing the third mode from fluttering via the $\omega$

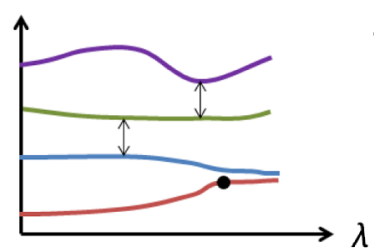

$g \uparrow$

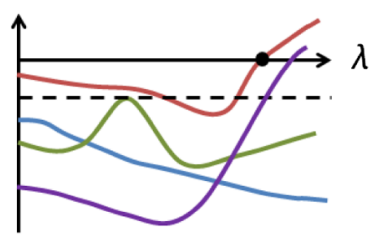

Fig. 4 Demonstration of frequency-separation constraints (left) and critical-damping constraints (right). hump-mode mechanism of Fig. $\underline{3}$, and the fourth mode from fluttering via the mechanism of Fig. 2 .

As before, however, imposition of these critical-damping constraints is found not to be sufficient: modal frequencies (which are not included in these constraints) could coalesce during the optimization process, leading to strong $C^{1}$ discontinuities in the $\lambda-g$ plot, and thus, in the design space as well. The obvious solution, used here, is to enforce both frequency-separation constraints and criticaldamping constraints. A drawback of this scheme is the large number of constraints that must be included in the optimization process. For a system with $N_{m}$ modes, $N_{m}-2$ frequency-separation constraints and $N_{m}-1$ critical-damping constraints must be maintained.

The feasible design space is fairly limited by the introduction of these constraints, which, as will be seen as follows, are aggressive and difficult for the optimizer to satisfy, that is, they have a large impact on the final topology. Presumably, an alternative handling of the eigenvalue-switching issues could provide superior optimal results. For stationary eigenvalue problems (i.e., buckling), several methods have been proposed, for example, a bound formulation [16] in conjunction with eigenvalue tracking. For eigenvalue-migration problems (i.e., aeroelastic flutter), alternatives to the aforementioned constraints are less obvious. This work demonstrates the topology optimization of blade-stiffened panels for buckling metrics using both separation constraints and bound techniques. For aeroelastic flutter problems, only separation constraints are demonstrated (specifically, the concepts of Fig. 4); this paper concludes with a discussion of alternative schemes.

\section{Numerical Formulation}

The stiffened-panel structures seen in Fig. 1 are discretized into quadrilateral facet-shell finite elements via a combination of linear strain triangles and discrete Kirchhoff triangles [17], in which two triangles form a single quadrilateral. A design variable, or density, $x_{e}$ is attached to each. This design variable is allowed to vary continuously between 0 (void) and 1 (solid), in which the SIMP [18] method is used. The linear stiffness matrix, geometric stiffness matrix, and mass matrix are computed by assembling over each finite element $(e)$ :

$$
\begin{gathered}
\boldsymbol{K}=\sum_{e}\left\{\boldsymbol{K}_{e} \cdot\left[x_{\min }+\left(1-x_{\min }\right) \cdot\left(x_{e}\right)^{p}\right]\right\} \\
\boldsymbol{K}_{\sigma}=\sum_{e}\left\{\boldsymbol{G}_{e}^{T} \cdot \boldsymbol{S}_{e}\left[\left(x_{e}\right)^{p}\right] \cdot \boldsymbol{G}_{e}\right\} \\
\boldsymbol{M}=\sum_{e}\left(\boldsymbol{M}_{e} \cdot x_{e}\right)
\end{gathered}
$$

in which $\boldsymbol{K}_{e}$ is an elemental stiffness matrix associated with solid elements, $x_{\min }$ is a small nonzero number, and $p$ is a penalization factor (typically greater than 2). $S_{e}$ is a matrix reordering of the element stress tensor [which is proportional to $\left(x_{e}\right)^{p}$ ], and $\boldsymbol{G}_{e}$ is a shape-function differentiation matrix [17]. Finally, $\boldsymbol{M}_{e}$ is the elemental mass matrix associated with solid elements.

The interpolation schemes used here are intended to prevent localized buckling and/or vibration modes in areas of low density [18]. It is further noted that, for this work, the prestresses in each finite element, $\boldsymbol{S}_{e}$, are from prescribed in-plane tractions within the face sheet. Future work may obtain these from a thermal-heat-conduction analysis. In keeping with the parameterization outlined in Fig. 1, only the topology within the stiffeners is allowed to change, and so $x_{e}$ for elements within the face sheet is fixed as unity. After the assembly of Eqs. (1-3), boundary conditions are applied, in which the perimeter of the face sheet is assumed to be simply supported. 


\section{Buckling-Eigenvalue Problems}

A buckling eigenproblem is defined as

$$
\left(\boldsymbol{K}+\beta_{n} \cdot \boldsymbol{K}_{\sigma}\right) \cdot \boldsymbol{\Phi}_{n}=\mathbf{0}
$$

in which $\boldsymbol{\Phi}_{n}$ is the eigenvector associated with the $n$th eigenvalue $\beta_{n}$, both of which will be real. As noted previously, $\beta_{n}$ is a scalar multiplier of the prescribed in-plane tractions within the face sheet. The topology-optimization problem considered here seeks to maximize the critical (first) buckling factor. This may be done by solving

$$
\max _{\tilde{\boldsymbol{x}}} \beta_{1} \quad \text { s.t.: } \begin{cases}0<x_{\min }<\tilde{x}_{e}<1 & e=1, \ldots, N_{e} \\ \boldsymbol{v}^{T} \cdot \boldsymbol{x} \leq V^{*} & \\ \beta_{n}-\beta_{n-1} \geq \varepsilon & n=2, \ldots, N_{m}\end{cases}
$$

in which the elemental design variables are grouped into the vector $\tilde{\boldsymbol{x}}$, which is then passed through a linearly decaying cone-shape filter [19] to obtain the variable densities $\boldsymbol{x}$. Side constraints, a volume constraint, and a series of eigenvalue separation constraints are also imposed. The eigenvalue sensitivities needed to solve Eq. (5) are readily computed; see [18] for details. The separation constraints stipulate that two consecutive eigenvalues be separated by some small amount $\varepsilon$, to prevent discontinuities associated with critical mode switching. This optimization problem (along with the others detailed as follows) may be efficiently solved with the method of moving asymptotes [20].

The eigenvalue separation constraints of Eq. (5) may overly restrict the design process. A better solution may be to remove the separation constraints entirely, and let mode switching occur in a smooth manner amenable to gradient-based optimization. As noted in the Introduction, this is readily accomplished for buckling problems (or any stationary eigenvalue problem). Equation (5) may be replaced with

$$
\max _{\tilde{\boldsymbol{x}}, b} b \quad \text { s.t.: } \begin{cases}0<x_{\min }<\tilde{x}_{e}<1 & e=1, \ldots, N_{e} \\ \boldsymbol{v}^{T} \cdot \boldsymbol{x} \leq V^{*} & n=1, \ldots, N_{m} \\ \beta_{n} \geq b & \end{cases}
$$

in which the bound $b$ is both an objective function and a design variable [16]. Mode tracking [21] may be employed to allow modes to cross (without being renumbered) as the optimization proceeds, and the likely scenario of bimodal eigenvalues $\left(\beta_{n}=\beta_{n+1}\right)$ may be accounted for as well [22]. It can be seen that Eq. (6) has one more constraint and one more design variable than Eq. (5) , but places no restraint on what the critical buckling mode must look like. Equation (5), on the other hand, requires that the identity of the critical buckling mode can never change, as modes cannot switch from their baseline ordering.

Results are given for a square aluminum panel (elastic modulus $E=70$ GPa modulus, density $\rho=2800 \mathrm{~kg} / \mathrm{m}^{3}$, and Poisson's ratio $\nu=0.3$ ), with a length $a$ of $0.3 \mathrm{~m}$ on each side, simply supported around the entire perimeter. For the entirety of this work, a supporting ground structure of three straight stiffeners is prescribed, each with a depth of $0.03 \mathrm{~m}$. Both the stiffeners and the face sheet have a spatially uniform thickness of $t=1.27 \mathrm{~mm}$. Each edge of the panel is discretized with 200 quadrilaterals, and 20 quadrilaterals are used depthwise through the stiffeners. The two outer stiffeners have the same topology, leaving 8000 total design variables within the vector $\tilde{\boldsymbol{x}}$. A compressive in-plane loading is applied to the panel, along the stiffener axis, to form $\boldsymbol{K}_{\sigma}$. Eigenvalue convergence histories are given in Fig. $\underline{5}$, in terms of a normalized load factor $N_{\mathrm{cr}}=\beta_{1} \cdot a^{2}$. $12 \cdot\left(1-\nu^{2}\right) /\left(\pi^{2} \cdot E \cdot t^{3}\right)$. The volume of the stiffeners is prescribed to be $10 \%\left(V^{*}\right)$ the volume of the face sheet for every case in this paper, although it should be noted that the convergence of the topology volume is not shown in Fig. 5 .

The left plot in Fig. $\underline{5}$ shows the convergence of Eq. (ㅁ), in which the critical buckling factor rapidly increases from the baseline value until the first separation constraint becomes active at iteration 15: $\beta_{1}-\beta_{2}=\varepsilon$, in which $\varepsilon$ is set (arbitrarily) to a normalized value of 0.4 . All four separation constraints are active by iteration 100 , and remain so through the end of the optimization process. The resulting topology (and its critical buckling mode shape) is shown in the upper right of Fig. 6 . The convergence of Eq. (6), contrastingly, proceeds much slower, and several mode crossings occur. The final optimal topology is seen in the lower left of Fig. 6, and eigenvalues 1, 3, and 5 (referencing the baseline numbering) are nearly equal to the bound $b$. The remaining two drawings in Fig. 6 show the eigensolution of an unstiffened panel $\left(V^{*}=0\right)$, and a fully stiffened panel $\left(x_{e}=1\right.$, $\left.V^{*}=0.3\right)$, for comparison purposes.

A common practice [18], used here, is to set the initial topology $\tilde{\boldsymbol{x}}$ equal to a spatially uniform value that exactly satisfies this volume constraint. For a $10 \%$ value, $\tilde{\boldsymbol{x}}$ is set to a uniform value of 0.333 , an intermediate density. This initial topology has a substantially higher buckling factor $\left(N_{\mathrm{cr}}=30.07\right)$ than the unstiffened panel $\left(N_{\mathrm{cr}}=\right.$ 4.00), but its buckling mode (which is not shown) is qualitatively similar to the unstiffened case. The fully stiffened panel, on the other hand, shows a higher-order mode shape (shorter wavelength). When

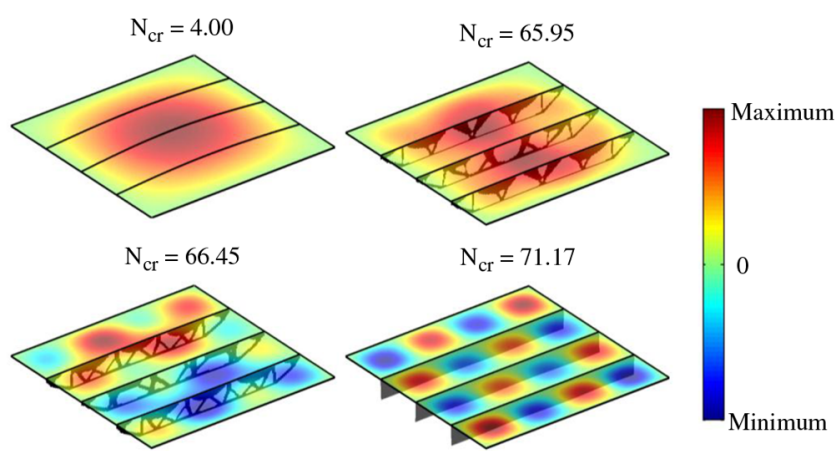

Fig. 6 Critical buckling factors and mode shapes for an unstiffened panel (upper left), a panel with optimized stiffeners using eigenvalue separation (upper right), a panel with optimized stiffeners using the bound method (lower left), and a fully stiffened panel (lower right).
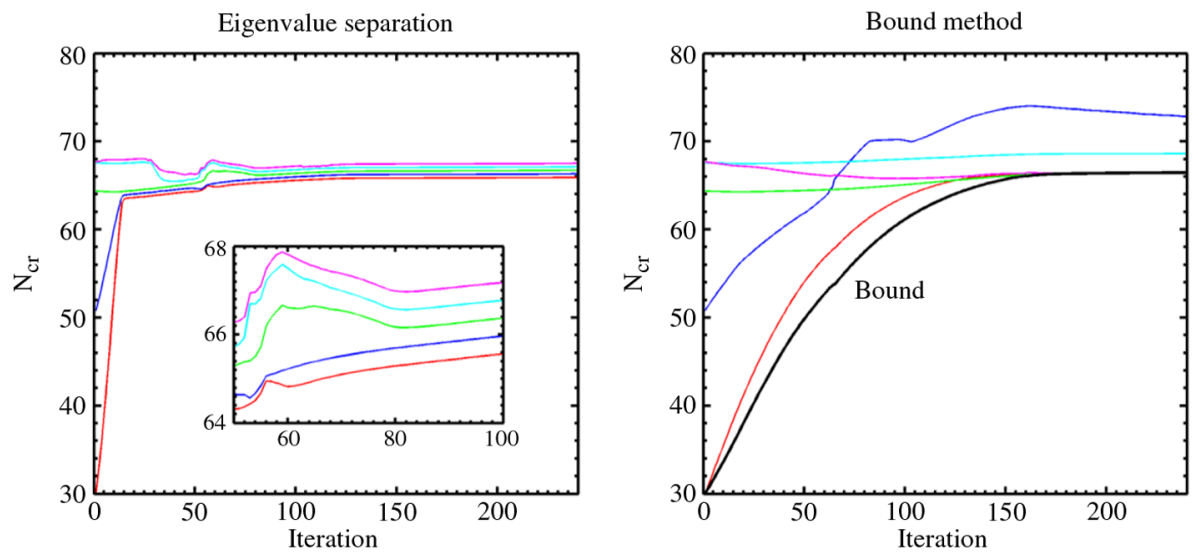

Fig. 5 Buckling-optimization convergence histories for eigenvalue separation-based methods [Eq. (므)] and bound formulations [Eq. (ㅁ)]. 
eigenvalue separation constraints are imposed [Eq. (5)], $\boldsymbol{\Phi}_{1}$ can never, during the course of the optimization process, look like the buckling mode of the fully stiffened panel. The imposition of the eigenvalue separation constraints prevents this from ever happening, as the identity of the critical mode can never change. The bound method [Eq. (6)] removes this requirement and results in a buckling mode, which shows some similarity to that of the fully stiffened panel. It should be noted, however, that this optimal topology has several closely-spaced modes. A mode shape which resembles the unstiffened panel's occurs at a buckling factor only $0.04 \%$ higher than that shown.

The expected result of Eq. (6) $\left(N_{\mathrm{cr}}=66.45\right)$, providing a superior optimum to Eq. (5) $\left(N_{\mathrm{cr}}=65.95\right)$, is achieved, but not by much. The optimal performance of the latter is a direct function of the chosen value for $\varepsilon$, which is set to some arbitrarily small value (0.4) for this work. Decreasing this value makes the constraint easier to satisfy, and will increase the optimal value of $N_{\mathrm{cr}}$ (and vice versa). If the value becomes too small, however, mode switching could still occur if the optimizer takes too large of a step, thus ultimately slowing down the convergence [14]. It is also noted that, although the connectivity between the upper and lower surfaces of each stiffener differs, the two optimal structures share many topological similarities. One may, thus, conclude that, for this specific problem, the choice between separation constraints and bound formulations is of minor consequence.

\section{Flutter-Eigenvalue Problems}

Next, a supersonic aeroelastic flutter eigenproblem is considered. This is an eigenvalue-migration problem, which is substantially more expensive than the stationary buckling-eigenvalue problems discussed previously. To alleviate the computational cost, flutter analyses are commonly [12] based on a reduced-order model. Freevibration modes of the stiffened panel are computed from

$$
\left(\boldsymbol{K}+\beta_{n} \cdot \boldsymbol{M}\right) \cdot \boldsymbol{\Phi}_{n}=0
$$

in which the eigenvalues in this case are $\beta_{n}=-\omega_{n}^{2}$, and $\omega_{n}$ are the natural vibration frequencies. Collecting the lowest few vibration modes into the matrix $\boldsymbol{\Phi}$, reduced matrices may be computed as $\boldsymbol{K}_{r}=\boldsymbol{\Phi}^{T} \cdot \boldsymbol{K} \cdot \boldsymbol{\Phi}$, and $\boldsymbol{M}_{r}=\boldsymbol{\Phi}^{T} \cdot \boldsymbol{M} \cdot \boldsymbol{\Phi}$. These new reduced matrices will be several orders of magnitude smaller than their fullorder counterparts (and diagonal as well), and are thus amenable to repeated eigenvalue calculations with increased values of $\lambda$ (as in Fig. 4). Two new problems are introduced, however.

First, several vibration modes are dominated by the motion of the stiffener, while the relative motion of the panel itself is much smaller. Airflow is assumed to travel over the upper surface of the panel only, and so does not explicitly interact with the stiffeners. Simply removing these modes degrades the accuracy of the flutter solution, because the vibration within the upper skin, although far smaller in magnitude than that seen in the stiffener, is still an important mode shape. Leaving these modes as is corrupts the generalized amplitude of each mode, owing to the large vibration of the stiffeners. An entirely ad hoc solution, used here, is to substructure the stiffness matrix into panel (1) and stiffener (2) degrees of freedom:

$$
\boldsymbol{K}=\left[\begin{array}{ll}
\boldsymbol{K}^{11} & \boldsymbol{K}^{12} \\
\boldsymbol{K}^{21} & \boldsymbol{K}^{22}
\end{array}\right]
$$

Upon the solution of Eq. (7), the stiffener degrees of freedom of each vibration mode are overwritten as

$$
\boldsymbol{\Phi}_{n}^{2}=-\left(\boldsymbol{K}^{22}\right)^{-1} \cdot \boldsymbol{K}^{21} \cdot \boldsymbol{\Phi}_{n}^{1}
$$

The final assembled mode shapes $\boldsymbol{\Phi}_{n}=\left\{\left(\boldsymbol{\Phi}_{n}^{1}\right)^{T} \quad\left(\boldsymbol{\Phi}_{n}^{2}\right)^{T}\right\}^{T}$ are then sent through a Gram-Schmidt process to orthogonalize the set. It is noted that, at the end of this process, neither $\boldsymbol{K}_{r}$ nor $\boldsymbol{M}_{r}$ is still diagonal.

A second issue arising from the use of a reduced-order model comes from the well-known issue of free-mode vs fixed-mode derivatives. Computing the analytical gradient of $\boldsymbol{K}_{r}$ or $\boldsymbol{M}_{r}$ with respect to the topological design variables $\tilde{\boldsymbol{x}}$ (to eventually compute the derivative of the flutter point) requires the derivative of $\boldsymbol{\Phi}$ as well. This is a very expensive term, due to the large number of structural degrees of freedom, large number of design variables, and (relatively) large number of mode shapes. For this work, these eigenvector gradients are simply omitted from the chain rule (fixed-mode derivatives), although mode shapes are updated at each new design point. Although this can, in some cases, provide fairly inaccurate gradients [23] , the optimizer is able to locate a well-defined topology under several active constraints, as will be seen as follows. Although exactly (or, more feasibly, approximately) accounting for these eigenvector derivatives may improve the convergence of the optimizer, it seems unlikely that the additional cost will be worthwhile.

An aeroelastic eigenvalue problem is written as

$$
\left(\left[\begin{array}{cc}
\mathbf{0} & -\boldsymbol{I} \\
\boldsymbol{K}_{r}-\lambda \cdot \boldsymbol{K}_{a} & \boldsymbol{C}_{r}-\sqrt{\lambda} \cdot \boldsymbol{C}_{a}
\end{array}\right]+\beta_{n} \cdot\left[\begin{array}{cc}
\boldsymbol{I} & \mathbf{0} \\
\mathbf{0} & \boldsymbol{M}_{r}
\end{array}\right]\right) \cdot \boldsymbol{\Phi}_{n}=\mathbf{0}
$$

in which $\boldsymbol{C}_{r}$ is a reduced structural Rayleigh damping matrix [17], $\boldsymbol{K}_{a}$ and $\boldsymbol{C}_{a}$ are aerodynamic stiffness and damping matrices computed via the supersonic piston theory (see [24] for derivation), and the dynamic pressure parameter (seen in Figs. 2-4) is defined as $\lambda=\rho_{\infty} \cdot U_{\infty}^{2} \cdot a^{3} \cdot 12 \cdot\left(1-\nu^{2}\right) /\left(E \cdot t^{3} \cdot \sqrt{M_{\infty}^{2}}-1\right)$, in which $\rho_{\infty}$, $U_{\infty}$, and $M_{\infty}$ are the density, speed, and Mach number of the flow traveling over the upper surface of the panel. $\boldsymbol{\Phi}_{n}$ is the right eigenvector associated with the $n$th eigenvalue $\beta_{n}$, both of which will be complex valued. Again, referencing Fig. $\underline{4}$, the eigenvalues are given by $\beta_{n}=g_{n} \pm i \cdot \omega_{n}$, in which only positive imaginary values are of interest. If $\lambda$ is set to 0 in Eq. (10) and Rayleigh damping is excluded, $g_{n}$ becomes zero, and the imaginary portions will coincide with the natural frequencies found in Eq. (7).

The composite damping of the system is the eigenvalue with the largest real part: $G=\max _{n}\left(g_{n}\right)$. The flutter point is the point in which $G=0$, and occurs at $\lambda=\lambda^{*}$. The topology-optimization problem is stated as

$$
\max _{\tilde{\boldsymbol{x}}} \lambda^{*} \quad \text { s.t.: } \begin{cases}0<x_{\min }<\tilde{x}_{e}<1 & e=1, \ldots, N_{e} \\
\boldsymbol{v}^{T} \cdot \boldsymbol{x} \leq V^{*} & \\
\left(\begin{array}{ll}
\min \\
0 \leq \lambda \leq \lambda^{*}
\end{array}\left(\omega_{n}-\omega_{n-1}\right)\right) \geq \varepsilon & n=3, \ldots, N_{m} \\
\left(\begin{array}{l}
\max \\
0 \leq \lambda \leq \lambda^{*}
\end{array}\left(g_{n}\right)\right) \leq g_{\text {cr }} & n=2, \ldots, N_{m}\end{cases}
$$

The frequency-separation constraints in Eq. (11) reflect the left part of Fig. 4, whereas the critical-damping constraints in Eq. (11) reflect the right part of Fig. 4 . Eigenvalues are traced as a function of $\lambda$ using the mode-tracking schemes developed in [21], specifically for nonself-adjoint systems. The precise flutter point $\lambda^{*}$ is computed using a direct eigenvalue-tracing method. A sensitivity analysis of $\lambda^{*}$, as well as critical eigenvalues (which may occur at any value of $\lambda$ between 0 and $\lambda^{*}$ ) dictated by frequency separation or critical damping, is readily available as well. The interested reader is referred to [25] for more details concerning these schemes.

For the flutter results, the panel geometry and material properties are unchanged, the Mach number $M_{\infty}$ is set to 10 , and the mass ratio $\rho_{\infty} \cdot a /(\rho \cdot t)$ is set to 2 . The results are given in Fig. 7, organized in a similar manner as the previous results. The flutter point $\lambda^{*}$, flutter frequency, and flutter mode shape are given, in which all frequencies have been normalized by the first natural frequency of an unstiffened panel. The flutter point $\lambda^{*}$ for the unstiffened panel agrees with the well-known result [26]. The optimal topology (again for a $10 \%$ volume fraction), as would be expected, is quite dissimilar from the buckling results. Very little connectivity is seen toward the trailing edge of the panel, despite the fact that most of the fluttering deformation occurs here. Unlike the buckling results, the fully 


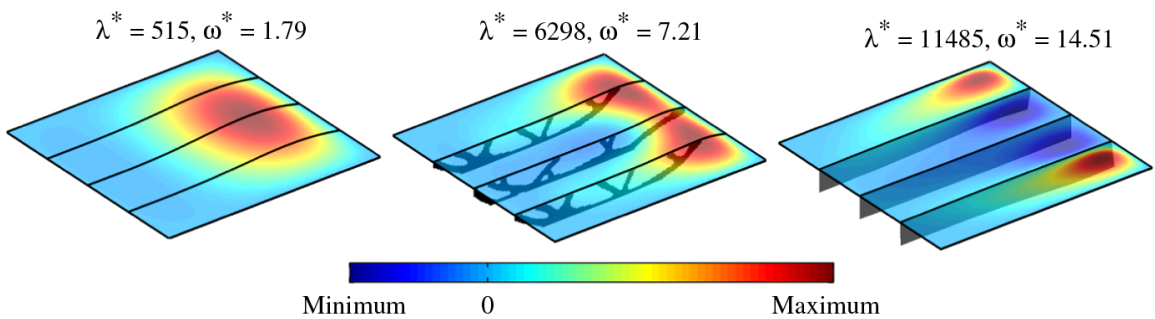

Fig. 7 Flutter points, frequencies, and mode shapes for an unstiffened panel (left), a panel with optimized stiffeners (center), and a fully stiffened panel (right).

stiffened panel substantially outperforms the optimal topology by nearly doubling the flutter speed (although the stiffeners are three times heavier). The tradeoff between the flutter and stiffener mass is far stronger than between the buckling and stiffener mass. Similar to the eigenvalue separation results of Fig. 6 (upper right), the flutter mode qualitatively resembles that of the unstiffened panel. A mode shape that resembles the fully stiffened panel (i.e., shorter wavelength, at least in the spanwise direction perpendicular to the stiffeners) is unattainable with the formulation of Eq. (12), due to the frequency-separation constraints. As before, the identity of the critical mode can never change. Flutter-analogous techniques to the bound method of Eq. (6) pose a number of complications, as will be discussed as follows.

The eigenvalue migration for the optimal topology of Fig. 7 is shown in Fig. 8, in which the first 10 modes are included in the analysis. Values of $\lambda$, which mark the point of lowest frequency separation between two consecutive modes, are given on the left of this figure. Only three of these frequency-separation constraints are active: between modes 2 and 3 (at a very low value of $\lambda=300$ ), between modes 4 and 5, and between modes 6 and 7 . These latter two separation points occur at the flutter point $\lambda^{*}$. The frequency separation between certain modes can become very small at values of $\lambda$ greater than the flutter point, but the constraint of Eq. (7) is only concerned with eigenvalue migration in the range $0 \leq \lambda \leq \lambda^{*}$. The separation value is set (again arbitrarily) as $\varepsilon=200 \mathrm{rad} / \mathrm{s}$, or a normalized value of 0.48 in the figure. As before, decreasing this value will make the constraint easier to satisfy, and should lead to a higher-performing optimum [25]. Decreasing it too much may cause inadvertent mode switching (if the optimizer takes too large of a step), and also may lead to strong $C^{1}$ discontinuities in the $\lambda-g$ plot if two frequencies nearly coalesce.

Similarly, points of critical damping are indicated on the right part of Fig. 8. Higher-order modes tend to be more stable at wind-off conditions $(\lambda=0)$, and therefore, have a lesser impact on the design process. This is a direct result of the choice made for the damping matrix $\boldsymbol{C}_{r}=\alpha_{c} \cdot \boldsymbol{M}_{r}+\beta_{c} \cdot \boldsymbol{K}_{r}$, in which $\alpha_{c}=20 \mathrm{~s}^{-1}$ and $\beta_{c}=10^{-4}$ s. If $\beta_{c}$ had been set to 0 , the wind-off damping of each mode would be equal, and thus, closer to the critical boundary. The critical-damping constraint is active, however $\left(g_{\text {cr }}=200 \mathrm{rad} / \mathrm{s}\right.$, or a normalized value of 0.48 in the figure), for lower modes 2,3 , and 4 ; a detailed graphic of this damping behavior near the flutter point is given in Fig. 9. The damping of modes 3 and 4 is equal to $g_{\mathrm{cr}}$ at $\lambda^{*}$; the ability of the optimizer to hold these two constraints prevents the type of mode-switching behavior drawn in Fig. 2 (with the potential fluttering of mode 4 the more aggressive of the two). Mode 2 is a hump mode, and is equal to $g_{\mathrm{cr}}$ slightly below the flutter point. Holding this constraint prevents the type of behavior drawn in Fig. 3.

A significant discrepancy between the optimal buckling topologies of Fig. 6 and the optimal flutter topology of Fig. 7 is the comparison between the performance of the optimal designs $\left(V^{*}=0.1\right)$ and the fully stiffened panels $\left(V^{*}=0.3\right)$. Despite the large reduction in stiffener mass, the buckling optimal topologies have only a mild drop in the buckling load; for the flutter problem, the tradeoff is far stronger. A direct comparison between the successes of the two design processes is perhaps unfair, given the completely different physical metrics under consideration. It seems likely, however, that the frequency-separation and (especially) the criticaldamping constraints used during flutter optimization are preventing the location of a superior design (even though the success of the buckling problem proved insensitive to the imposition of these constraints). As noted previously, one key is to allow the optimizer to

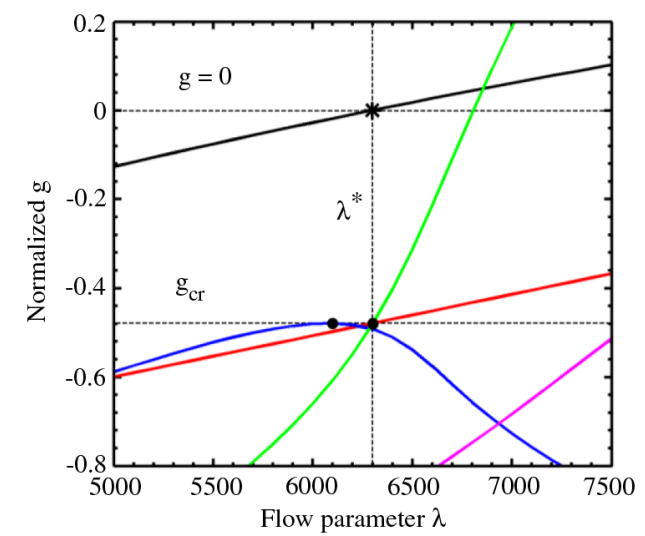

Fig. 9 Eigenvalue (real part) migration close to the flutter point of the optimal topology in Fig. 7 .
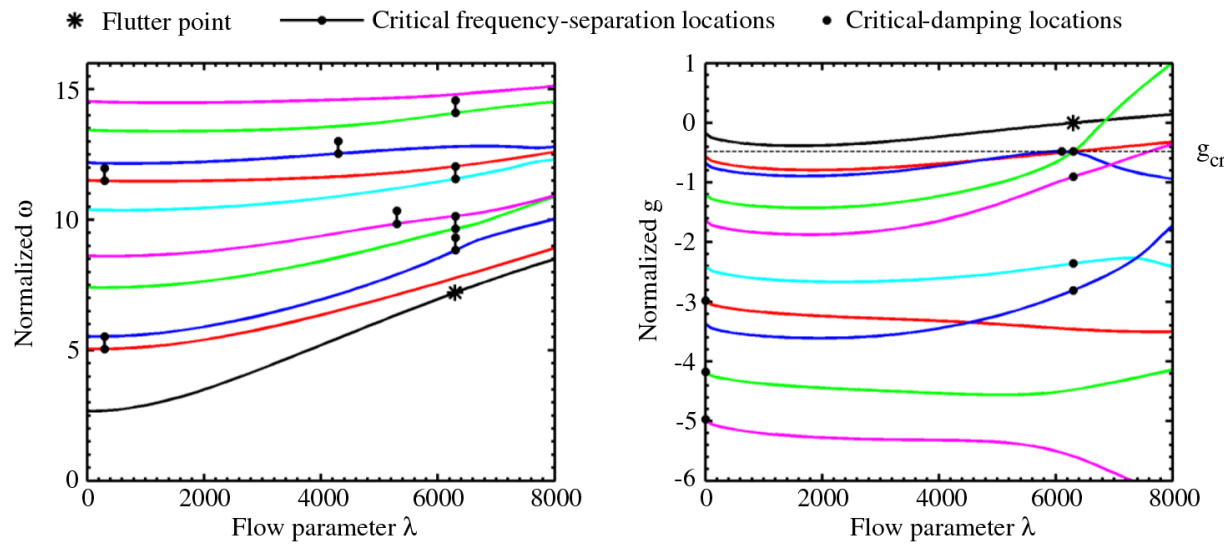

Fig. 8 Eigenvalue migration of the optimal topology in Fig. 7. 

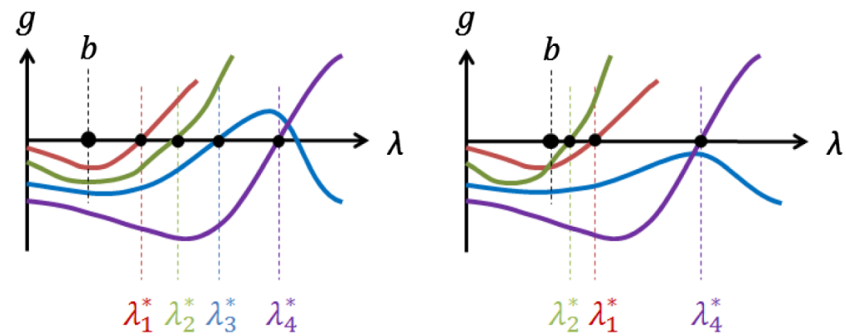

Fig. 10 Eigenvalue migration (real part): tracking multiple flutter mechanisms from one design iterate (left) to the next (right).

transition from a simple panel mode shape $\boldsymbol{\Phi}_{1}$ (left of Fig. 7) to one with a shorter wavelength (right of Fig. 7), as the latter will typically have a higher $\lambda^{*}$. The frequency-separation constraints of Eq. (11) clearly prevent this in their goal of providing a smooth design space (i.e., no mode switching).

One ad hoc solution would be to impose an initial uniform value of $\tilde{\boldsymbol{x}}$, which violates the volume constraint $V^{*}$, but provides enough stiffness such that $\boldsymbol{\Phi}_{1}$ is qualitatively similar to the flutter mode of the fully stiffened panel. A better solution would be to remove the separation constraints entirely, and let mode switching occur in a smooth manner amenable to gradient-based optimization. A flutteranalogous version of the bound method is written as

$$
\max _{\tilde{\boldsymbol{x}}, b} b \quad \text { s.t. } \begin{cases}0<x_{\min }<\tilde{x}_{e}<1 & e=1, \ldots, N_{e} \\ \boldsymbol{v}^{T} \cdot \boldsymbol{x} \leq V^{*} & \\ \lambda_{n}^{*} \geq b & n=1, \ldots, N_{m}\end{cases}
$$

This equation is essentially the same as Eq. (6), substituting $\beta_{n}$ in the last constraint with $\lambda_{n}^{*}$. This new term $\lambda_{n}^{*}$ represents successive flutter points, as drawn in Fig. 10. In moving from one hypothetical design iterate (left plot) to the next (right plot), the first two flutter points switch, with $\lambda_{2}^{*}$ the new critical flutter point. The two points are not renumbered, as mode tracking [21] can again be used to place a distinction between the modes beyond mere ordering. Both $\lambda_{1}^{*}$ and $\lambda_{2}^{*}$ are being pushed to higher values in these plots by an increase in the bound value $b$.

With regard to flutter problems, one important drawback of this scheme is the significantly increased computational cost. Equation (11) only requires the location of a single flutter point, whereas Eq. (12) requires the location of several. The piston-theory aerodynamics used here has the luxury of explicit state-space aerodynamics $\left(\boldsymbol{K}_{a}, \boldsymbol{C}_{a}\right)$, but many aerodynamic theories only exist in the frequency domain [27] (doublet-lattice methods, or many computational fluid dynamics-based tools used for eigenvalue-based flutter interrogation), and so the solution of Eq. (10) must proceed in a tedious and expensive iterative manner. With these methods, only computing the first flutter point $\lambda_{1}^{*}$ can be expensive in itself. Furthermore, the advent of hump modes during the optimization process (or their disappearance, as demonstrated with $\lambda_{3}^{*}$ in Fig. 10) will also cause significant issues with Eq. (12), as the number of flutter points $N_{m}$ will change, and thus, the number of constraints will also. This occurs during the development of the optimal structure in Fig. 7; the hump mode of Fig. 9 is not present in the initial baseline design. The aforementioned oscillatory aerodynamics (written in the frequency domain) are known [27] to have entire modes suddenly appear or disappear with increased $\lambda$, which will further stress an algorithm like Eq. (12).

Future work may resolve these issues, as well as demonstrate a cost-performance tradeoff between optimal topologies found via Eqs. (11) and (12) [or a version of Eq. (12), which can handle the noted mode-bifurcation issues]. Future work must also account for the problems of local mode vibration (when constructing a reduced basis $\boldsymbol{\Phi}$ ), and fixed- vs free-mode derivatives (accounting for the gradient of $\boldsymbol{\Phi}$ with respect to the design variables). Both issues were resolved with simple ad hoc methods for this work, although more fundamental and robust solutions are obviously desirable.

\section{Conclusions}

This work presents the topology optimization of stiffened panels subject to buckling and aeroelastic flutter metrics. A square metallic skin, simply supported along its perimeter, is subject to in-plane loads (to quantify buckling resistance), or to supersonic flow over its upper surface (to quantify the flutter point). A network of stiffeners is attached to the underside of the panel, discretized into a lattice of finite elements, and SIMP-based topology optimization is used to find the best layout of material within each. This paper restricts this network to three straight blade stiffeners, although future work may look at a finer and more complex ground structure, or find the ground structure as the result of a prior optimization.

The consideration of buckling and flutter metrics constitutes an eigenvalue-optimization problem. This class of problem poses several challenges, the most significant of which is the crossover of eigenvalue roots with changes in the design variables. If left unhandled, this behavior can lead to a discontinuous design space, resulting in a slow (or complete lack of) convergence of the gradientbased optimizer. For buckling problems, two methods are demonstrated via constraints that, if satisfied, will enforce a certain level of smoothness on the design behavior. The first imposes a buffer between two consecutive eigenvalues, whereas the second is a standard bound method (which allows modes to freely migrate during the optimization). For flutter problems, only the first method is implemented: separation constraints are applied to both the real (damping) and the imaginary (frequency) parts of each eigenvalue. These constraints, although they ensure a smooth design space, also impose a lock-in behavior of modes during the optimization process, so that the first mode will always remain the critical mode, and no mode crossover is allowed. Although this method performs well in comparison to the bound method for buckling problems, it may be too restrictive for flutter problems. Unfortunately, a flutter-analogous bound method is beset with several complicating issues, each of which is discussed and may be considered in future work.

\section{Acknowledgment}

This work is sponsored by the U.S. Air Force Office of Scientific Research under laboratory task 03VA01COR (monitored by Fariba Fahroo).

\section{References}

[1] Bedair, O., "Analysis and Limit State Design of Stiffened Plates and Shells: A World View," Applied Mechanics Reviews, Vol. 62, No. 2, 2009, Paper 020801.

doi: $10.1115 / 1.3077137$

[2] Bhatia, M., Kapania, R., and Evans, D., "Comparative Study on Optimal Stiffener Placement for Curvilinearly Stiffened Panels," Journal of Aircraft, Vol. 48, No. 1, 2011, pp. 77-91. doi:10.2514/1.C000234

[3] Kim, W., Grandhi, R., and Haney, M., "Multiobjective Evolutionary Structural Optimization Using Combined Static/Dynamic Control Parameters," AIAA Journal, Vol. 44, No. 4, 2006, pp. 794-802. doi:10.2514/1.16971

[4] Luo, J., and Gea, H., "A Systematic Topology Optimization Approach for Optimal Stiffener Design,” Structural Optimization, Vol. 16, No. 4, 1998, pp. 280-288.

doi:10.1007/BF01271435

[5] Lam, Y., and Santhikumar, S., "Automated Rib Location and Optimization for Plate Structures," Structural and Multidisciplinary Optimization, Vol. 25, No. 1, 2003, pp. 35-45. doi:10.1007/s00158-002-0270-7

[6] Khosravi, P., Sedaghathi, R., and Ganesan, R., "Optimization of Stiffened Panels Considering Geometric Nonlinearity," Journal of Mechanics of Materials and Structures, Vol. 2, No. 7, 2007, pp. 12491265.

doi:10.2140/jomms.2007.2.1249

[7] Ding, X., and Yamazaki, K., "Stiffener Layout Design for Plate Structures by Growing and Branching Tree Model (Application to Vibration-Proof Design)," Structural and Multidisciplinary Optimization, Vol. 26, Nos. 1-2, 2004, pp. 99-110. doi:10.1007/s00158-003-0309-4 
[8] Bojczuk, D., and Szteleblak, W., "Optimization of Layout and Shape of Stiffeners in 2D Structures," Computers \& Structures, Vol. 86, Nos. 13-14, 2008, pp. 1436-1446. doi:10.1016/j.compstruc.2007.05.005

[9] Pedro, H., and Kobayashi, M., "On a Cellular Division Method for Topology Optimization," International Journal for Numerical Methods in Engineering, Vol. 88, No. 11, 2011, pp. 1175-1197. doi:10.1002/nme.v88.11

[10] Maute, K., and Ramm, E., "Adaptive Topology Optimization of Shell Structures," AIAA Journal, Vol. 35, No. 11, 1997, pp. 1767-1773. doi: $10.2514 / 2.25$

[11] Stegmann, J., and Lund, E., "Nonlinear Topology Optimization of Layered Shell Structures," Structural and Multidisciplinary Optimization, Vol. 29, No. 5, 2005, pp. 349-360. doi:10.1007/s00158-004-0468-y

[12] Bisplinghoff, R., Ashley, H., and Halfman, R., Aeroelasticity, Addison Wesley, Cambridge, MA, 1955.

[13] Langthjem, M., and Sugiyama, Y., "Optimum Shape Design Against Flutter of a Cantilevered Column with an End-Mass of Finite Size Subjected to a Non-Conservative Load," Journal of Sound and Vibration, Vol. 226, No. 1, 1999, pp. 1-23. doi:10.1006/jsvi.1999.2211

[14] Odaka, Y., and Furuya, H., "Robust Structural Optimization of Plate Wing Corresponding to Bifurcation in Higher Mode Flutter," Structural and Multidisciplinary Optimization, Vol. 30, No. 2, 2005, pp. 437-446. doi:10.1007/s00158-005-0538-9

[15] Haftka, R., "Parametric Constraints with Application to Optimization for Flutter Using a Continuous Flutter Constraint," AIAA Journal, Vol. 13, No. 4, 1975, pp. 471-475. doi: $10.2514 / 3.49733$

[16] Olhoff, N., "Multicriteria Structural Optimization via Bound Formulation and Mathematical Programming," Structural and Multidisciplinary Optimization, Vol. 1, No. 1, 1989, pp. 11-17. doi:10.1007/BF01743805

[17] Cook, R., Malkus, D., Plesha, M., and Witt, R., Concepts and Applications of Finite Element Analysis, Wiley, New York, 2002.
[18] Bendsøe, M., and Sigmund, O., Topology Optimization, Springer, Berlin, 2003.

[19] Bruns, T., and Tortorelli, D., "Topology Optimization of Non-Linear Elastic Structures and Compliant Mechanisms," Computer Methods in Applied Mechanics and Engineering, Vol. 190, Nos. 26-27, 2001, pp. 3443-3459. doi:10.1016/S0045-7825(00)00278-4

[20] Svanberg, K., "The Method of Moving Asymptotes-A New Method for Structural Optimization," International Journal for Numerical Methods in Engineering, Vol. 24, No. 2, 1987, pp. 359-373. doi:10.1002/(ISSN)1097-0207

[21] Eldred, M., Venkaya, V., and Anderson, W., "New Mode Tracking Methods in Aeroelastic Analysis," AIAA Journal, Vol. 33, No. 7, 1995, pp. 1292-1299. doi: $10.2514 / 3.12552$

[22] Seyranian, A., Lund, E., and Olhoff, N., "Multiple Eigenvalues in Structural Optimization Problems," Structural Optimization, Vol. 8, No. 4, 1994, pp. 207-227. doi:10.1007/BF01742705

[23] Haftka, R., and Gürdal, Z., Elements of Structural Optimization, Kluwer, Dordrecht, The Netherlands, 1992.

[24] Stanford, B., and Beran, P., "Computational Strategies for ReliabilityBased Structural Optimization of Aeroelastic Limit Cycle Oscillations," Structural and Multidisciplinary Optimization, Vol. 45, No. 1, 2012, pp. 83-99. doi:10.1007/s00158-011-0663-6

[25] Stanford, B., and Beran, P., "Aerothermoelastic Topology Optimization with Flutter and Buckling Metrics," Structural and Multidisciplinary Optimization, Vol. 48, No. 1, 2013, pp. 146-171. doi: $10.1007 / \mathrm{s} 00158-013-0885-\mathrm{x}$

[26] Dowell, E., "Nonlinear Oscillations of a Fluttering Plate," AIAA Journal, Vol. 4, No. 7, 1966, pp. 1267-1275. doi: $10.2514 / 3.3658$

[27] van $\mathrm{Zyl}, \mathrm{L}$. , and Maserumule, M., "Divergence and the $p-k$ Flutter Equation," Journal of Aircraft, Vol. 38, No. 3, 2001, pp. 584-586. doi: $10.2514 / 2.2805$ 